\title{
Nonwoven Hemp Fibre Reinforced Acrodur Biocomposites and their Mechanical Performance in Immersed Water
}

ISSN: 2576-8840

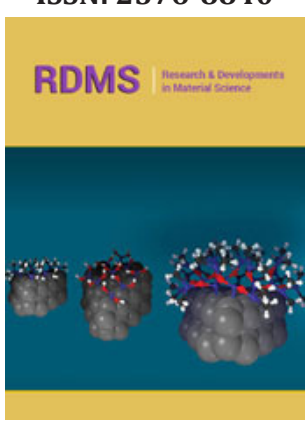

*Corresponding author: Islam MS, School of Mechanical and Manufacturing Engineering, The University of New South Wales, Australia

Submission: 覀 November 09, 2020

Published: N November 20, 2020

Volume 14 - Issue 3

How to cite this article: Islam MS, MiaoM. Nonwoven Hemp Fibre Reinforced Acrodur Biocomposites and their Mechanical Performance in Immersed Water. Res Dev Material Sci. 14(3). RDMS.000838. 2020. DOI: 10.31031/RDMS.2020.14.000838

Copyright@ Islam MS. This article is distributed under the terms of the Creative Commons Attribution 4.0 International License, which permits unrestricted use and redistribution provided that the original author and source are credited.
Islam MS ${ }^{1,2 *}$ and Miao $\mathbf{M}^{2}$

${ }^{1}$ School of Mechanical and Manufacturing Engineering, The University of New South Wales, Australia

${ }^{2}$ CSIRO Materials Science and Engineering, Australia

\begin{abstract}
Acrodur, aqueous polyacrylic acid solution and dispersion, has been developed as an alternative wood adhesive to phenolic and urea formaldehyde resins. It has many environmental benefits over the conventional thermoset resins that include its non-corrosive nature and non-emission of carcinogenic gases. It has superior tolerance to moisture during composite fabrication and thus drying of the preforms may be minimised or even eliminated contrary to most resins used in natural fibre biocomposites. The aim of this study was to produce optimised hemp fibre reinforced Acrodur resin biocomposites using nonwoven hemp fibre mats by varying Acrodur solution to dispersion ratio, relative humidity, curing time and temperature. The optimised biocomposites gave the best combination of $26.4 \mathrm{MPa}-\mathrm{cm} 3 / \mathrm{g}$ specific tensile strength and $4.4 \mathrm{GPa}-\mathrm{cm} 3 / \mathrm{g}$ specific Young's modulus with $0.94 \mathrm{~g} / \mathrm{cm} 3$ density. Thermogravimetric analysis (TGA) and contact angle measurement showed similar thermal stability and increased hydrophobicity of the biocomposites than those of the fibres. From the water immersion test, the path of wicking of water moleculesinto the biocomposites was believed to be rather straight than tortuous and about $43.5 \%$ loss of tensile strength and $57.1 \%$ loss of Young's modulus were observed upon 4-week water immersion of the biocomposites.
\end{abstract}

Keywords: Polymer-Matrix Composites (PMCs);Mechanical testing; Fibres; Compression moulding

Introduction

The use of natural plant fibres such as flax, hemp, kenaf, jute and wood fibres as reinforcement in both thermoplastics and thermosets has increased dramatically during the last twenty years. These natural fibre biocomposites compare well with glass fibre reinforced polymer composites in terms of recyclability when using a thermoplastic matrix and energy recovery through incineration when using a thermoset matrix, and with traditional structural materials in terms of specific mechanical properties [1]. Thermoset polymers are particularly attractive as matrix materials for natural plant fibre reinforced biocomposite production as they generally have reactive functional groups that make them compatible with hydrophilic fibre surfaces [1].

Hemp is one of the strongest and stiffest available renewable natural fibres which consists mainly of crystalline cellulose (55-72 wt\%) as well as hemicellulose (8-19\%), lignin (2-5\%) and waxy substances [2].

Conventional thermoset matrices such as polyester and vinyl ester have the ability to reinforce natural fibres. However, polyester and vinyl ester matrices emit styrene not only during processing but also from the finished product. Styrene is classified as a possible carcinogen by the International Agency for Research on Cancer (IARC) and known as a photochemical ozone creator. This ozone is highly reactive and is known to affect human health, crops, forests. Other thermoset resins such as resole type phenolic resins (phenolformaldehyde) and urea-formaldehyde resins are used extensively in the production of wood composite board [3,4]. Both of these resins possess exceptionally good adhesive properties, high rigidity and dimensional stability. Phenolic resins have exceptional heat and fire resistance due to their highly crosslinked aromatic structures and urea-formaldehydes 
have the ability to glue oily woods. However, both phenol and urea formaldehydes emit formaldehyde which is also classified as a carcinogen for humans [5,6]. Formaldehyde also causes eye, nose, and respiratory irritation and can be an asthma trigger [7] during composites production by hot pressing and from the finished composite boards. Therefore, the use of these common thermoset resins as binders is not engaging. Researchers around the world are trying to find alternative binders.

Acrodur is a polyester of polycarboxylic acid and polyalcohol and is an aqueous formaldehyde free binder system with high heat resistance and excellent storage stability. Acrodur is produced in two forms (a) Acrodur solutions and (b) Acrodur dispersions.
Acrodur solutions are made from a polycarboxylic acid with a polyalcohol dissolved in water. Acrodur dispersions are made from a polycarboxylic acid modified with a latex component along with a polyalcohol dissolved in water (Figure 1). Acrodur solutions can be mixed with Acrodur dispersions to produce properties ranging from highly flexible to highly stable duroplastic. Acrodur binders are readily miscible with water and conventional polymer binders and additives, allowing simple, precise and fine-tuning of the processing parameters to obtain desired final properties. Examples of the versatile range of applications for Acrodur include wooden and natural fibre mouldings for the automotive industry, abrasives from corn cob, and cellulose substrate-based filter paper for air and oil filters.

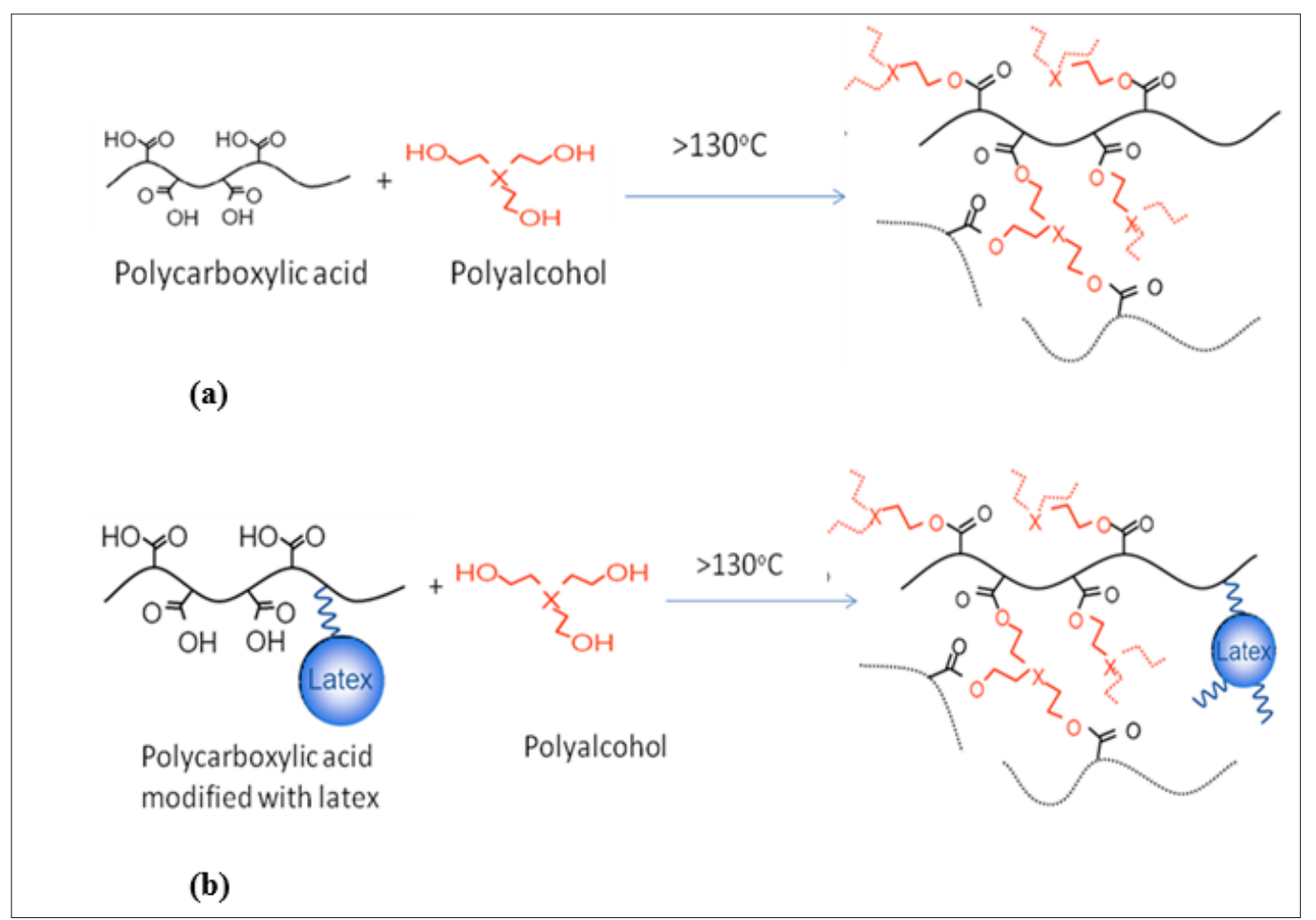

Figure 1: (a) Acrodur solution and (b) Acrodur dispersion formation reactions.

Acrodur offers significant benefits such as diversity, ease of processing, and high environmental compatibility due to its noncorrosive properties. It produces only one by-product during thermal curing reaction: water. It has low density and Acrodur solution can be mixed with Acrodur dispersion to modify its mechanical and water absorption properties. Besides these, Acrodur binders are very easy to handle and can be applied to a wide range of substrates using any of the commonly used techniques. The ingredients in Acrodur do not require special filters or environmental protection measures. Moreover, Acrodur is believed to have the ability to form hydrogen and covalent bonds with the hydroxyl groups present in cellulose of natural plant fibres through ester groups.

The objective of this study was to investigate the effect of Acrodur solution to dispersion ratio, relative humidity of nonwoven hemp fibre mat and curing temperature and time on the mechanical properties of nonwoven hemp fibre Acrodur biocomposites (HAB). The thermal degradation, hydrophobicity of the optimised biocomposites and ageing of the biocomposites upon immersion in water were also studied.

\section{Experimental}

\section{Materials}

Acrodur solution (Acrodur 950L) with a density of $1.06 \mathrm{~g} / \mathrm{cm}^{3}$ and molecular weight of ca $80,000 \mathrm{~g} / \mathrm{mol}$ and Acrodur dispersion (DS 3515) with a density of $1.20 \mathrm{~g} / \mathrm{cm}^{3}$, molecular weight of ca $25,000 \mathrm{~g} / \mathrm{mol}$ and latex particle size of ca $80 \mathrm{~nm}$ were obtained from BASF, Germany. Hemp fibre in the form of random non-woven mat was obtained from Canada. 


\section{Methods}

Differential scanning calorimetry: A DSC 7 differential scanning calorimeter from Perkin Elmer was used to monitor the exothermic thermograms of isothermal DSC measurements. A steady isothermal baseline was established at the preset cure temperature using two empty sample aluminium pans. Acrodur solution to Acrodur dispersion ratio was 3:1 for all the measurements. The specimens were enclosed in a closed aluminium container to prevent any evaporation and were scanned while keeping a static air flow of $50 \mathrm{~mL} / \mathrm{min}$. Isothermal scans were carried out at 160, 180 and 200 ${ }^{\circ} \mathrm{C}$ and the curing reactions were considered to be completed when the isothermal DSC thermograms levelled off to the baseline. The weight of each specimen was approximately $10 \mathrm{mg}$.

\section{Fabrication of biocomposites}

A. HAB produced with acrodur solution, acrodur dispersion and a mixture of acrodur solution and dispersion

Nonwoven hemp fibre was cut into $30 \mathrm{~cm} \times 30 \mathrm{~cm}$ mats using a hydraulic cutter (Torielli, Vigevano-Italia) and dried in an oven at $80{ }^{\circ} \mathrm{C}$ until the weight was stabilised (i.e., evaporation completed). A mixture of Acrodur solution to dispersion at the ratio of 3:1 was prepared. The dried hemp mats were then hand laid-up with the Acrodur solution, Acrodur dispersion and mixture of Acrodur solution and dispersion respectively and were subsequently pressed into biocomposites using a compression mould at a curing temperature of $160{ }^{\circ} \mathrm{C}$ for $20 \mathrm{~min}$.

B. HAB produced at different acrodur solution to dispersion ratio

Three mixtures of Acrodur solution to dispersion at ratios 3:1, 3:3 and 1:1 was prepared. The dry hemp fibre mat was laid with three different Acrodur mixtures respectively and subsequently pressed at a curing temperature of $160{ }^{\circ} \mathrm{C}$ for 20 minutes.

\section{HAB produced at different curing temperature and} time

The hand lay-ups prepared according to the above procedure were pressed into biocomposites at three different curing temperatures 160,180 and $200{ }^{\circ} \mathrm{C}$ for 20,10 and 5 minutes, respectively.

\section{HAB produced at different relative humidities}

The hemp fibre mats were cut and dried according to the above procedures and placed in a humidity chamber set to a constant temperature of $25^{\circ} \mathrm{C}$ and required relative humidity (RH) levels for 72 hours. The relative humidity levels used were 40,60 and $80 \%$. The dry fibre mat and the mats conditioned to 40,60 and $80 \% \mathrm{RH}$ were hand laid-up with an Acrodur solution/dispersion mixture (ratio 3:1) and subsequently pressed into biocomposites using a compression mould at a curing temperature of $160^{\circ} \mathrm{C}$ for $20 \mathrm{~min}$.

The fibre content of all of the biocomposites produced by (a), (b), (c) and (d) was found to be approximately $60 \mathrm{wt} \%$.

\section{Digital and optical microscopy}

The porous surface of the cured Acrodur was photographed using a Sony digital camera (model: cyber-shot DSC-HX20V). The surfaces of the control and water immersed biocomposite specimens were examined using an optical microscope (Olympus BX 60) with a calibrated eyepiece at 200x magnification to assess biocomposite surface porosity and separated fibres upon degradation by water uptake.

\section{Tensile testing}

The biocomposites produced were cut into tensile test specimens in both machine (warp) and cross-machine (weft) direction of the production of nonwoven hemp fibre mats using a scroll saw in accordance with ASTM D 638-03 standard test method for Tensile Properties of Plastics and placed in a conditioning chamber at 23 ${ }^{\circ} \mathrm{C} \pm 3{ }^{\circ} \mathrm{C}$ and $50 \% \pm 5 \% \mathrm{RH}$ for $24 \mathrm{hrs}$. The specimens were then tested using an INSTRON 5500R tensile testing machine fitted with a $100 \mathrm{kN}$ load cell at a rate of $5 \mathrm{~mm} / \mathrm{min}$. Five to six specimens were used for each test and tested up to the point of failure.

\section{Thermogravimetric analysis}

Thermogravimetric Analysis (TGA) of hemp fibre, cured Acrodur resin and HAB was carried out using a Shimadzu TGA-60 instrument. All the measurements were taken whilst maintaining a static air flow of $150 \mathrm{~mL} / \mathrm{min}$ with a constant heating rate of $10{ }^{\circ} \mathrm{C}$ $/$ min in an open alumina crucible. The weight of the specimens was around $10 \mathrm{mg}$, with a scanned temperature range from 25 to $600{ }^{\circ} \mathrm{C}$.

\section{Contact angle measurement}

Contact angle measurements were conducted using KSV CAM100 contact angle and surface tension tester. The droplet used was distilled water and was 180 pixels in size. Five specimens from each sample were tested. For each measurement a $15 \mathrm{~mm}$ square sample was cut from the nonwoven hemp fibre mat, cured Acrodur resin and $\mathrm{HAB}$ and mounted onto a glass slide using double sided tape. Specimens were cut from random areas of the samples.

\section{Water immersion tests}

Water immersion testing of the samples (biocomposites produced with dry nonwoven hemp fibre mat at Acrodur solution to dispersion ratio of $3: 1$ and cured at $160{ }^{\circ} \mathrm{C}$ ) were carried out in accordance with ASTM D 570-98: Standard Test Method for Water Absorption of Plastics for up to 4 weeks. The samples were submerged in water at $25{ }^{\circ} \mathrm{C}$ and removed from the water every 1 week interval, wiped with a clean dry cloth to remove surface water, and wiped with paper towel to remove water from the pores, weighed in a high precision balance to assess the weight change and then re-submerged for continued ageing. Tensile testing of the aged samples was carried out in accordance with ASTM standards as described in section 4.2.3. Five specimens of each batch were tested. 


\section{Results and Discussion}

\section{Differential scanning calorimetry measurements of acrodur resin}

Figure 2 shows a typical heat flow versus time curve for the Acrodur mixture (Acrodur solution to dispersion ratio of 3:1) cured at $200{ }^{\circ} \mathrm{C}$. The Acrodur mixture curve levels off the base line in around five minutes. Acrodur mixture at three curing temperatures showed similar pattern but different time scales. The time required to cure the Acrodur mixture at these three different isothermal curing temperatures of 160,180 and $200^{\circ} \mathrm{C}$ was 20,10 and 5 minutes, respectively. As expected, the time required for curing was found to decrease with the increase of temperature.

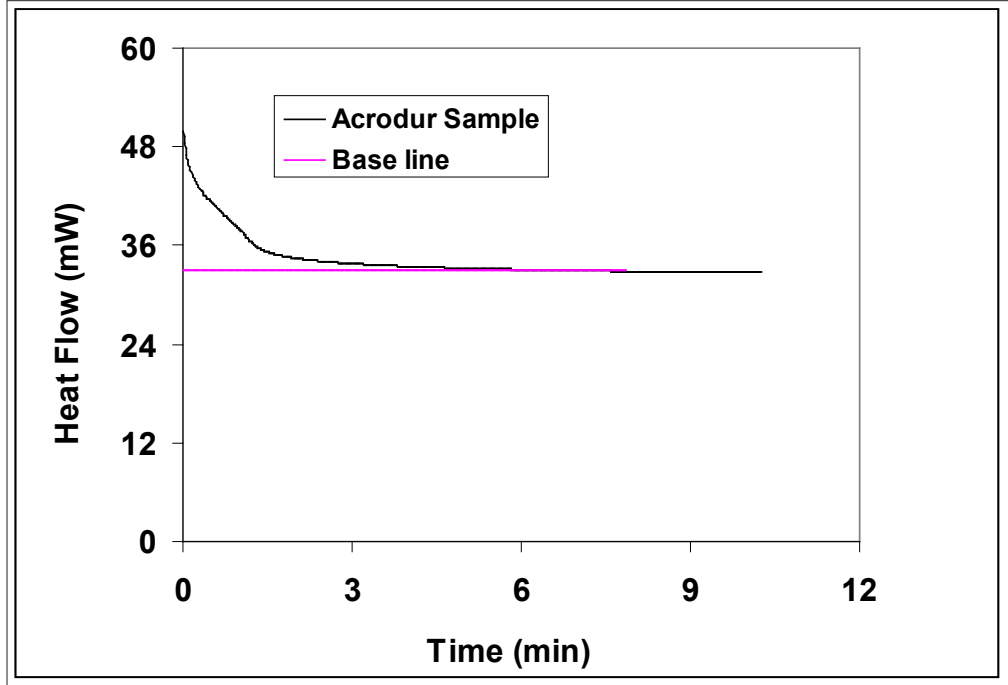

Figure 2: A typical heat flow versus time curve for the curing of an Acrodur mixture (Acrodur solution to dispersion ratio of $3: 1$ ) at $200{ }^{\circ} \mathrm{C}$.

\section{Tensile properties of the biocomposites}

HAB produced with acrodur solution, acrodur dispersion and different acrodur solution to dispersion ratio: Figure 3(a) \& 3(b) shows the tensile strength and Young's modulus of HAB produced with Acrodur solution, Acrodur dispersion and three different mixtures of Acrodur solution and dispersion at the ratios of $3: 1,3: 2$ and $1: 1$. All of the biocomposites produced here were tested in machine (warp) direction of the nonwoven hemp fibre mat. From the results of the biocomposites, it can be seen that the average specific tensile strength is the highest for the biocomposites produced with an Acrodur solution to dispersion ratio of 3:1 followed by Acrodur solution to dispersion ratio of $3: 2$, Acrodur solution to dispersion ratio of $1: 1$, Acrodur solution and finally Acrodur dispersion. In the case of specific Young's modulus, the highest value of 4.4GPa was seen for Acrodur solution to dispersion ratio of 3:1 which was followed by Acrodur solution, Acrodur solution to dispersion ratio of 3:2, Acrodur solution to dispersion ratio of 1:1, and finally Acrodur dispersion. Therefore, it can be conferred that the optimised proportion for the HAB would be Acrodur solution to dispersion ratio of 3:1 which might give optimised distribution and dispersion of Acrodur to form hydrogen and covalent bond with acid group of Acrodur and hydroxyl group of hemp fibre.

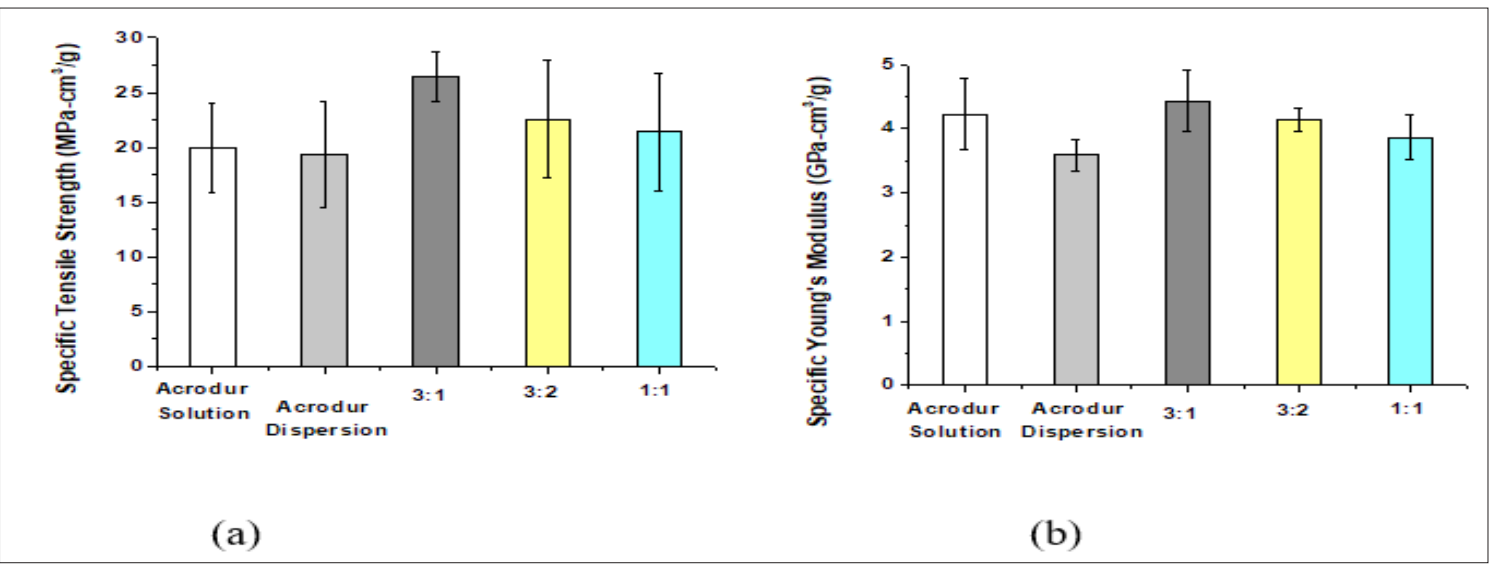

Figure 3: Tensile properties of HAB produced with Acrodur solution, Acrodur dispersion and three different Acrodur solution to dispersion ratios. 
HAB produced at different curing temperature and time: In the investigation of the optimization of processing conditions of the $\mathrm{HAB}$, they were produced with Acrodur solution to dispersion ratio of 3:1 at three different curing temperatures and time. The curing time was taken from the corresponding curing temperatures of the DSC scans as described in section 5.1. From the results of the biocomposites (Figures 4(a) \& 4(b)), both average tensile strength and Young's modulus were highest for the biocomposites produced at $160{ }^{\circ} \mathrm{C}$ followed by 180 and $200{ }^{\circ} \mathrm{C}$. The decrease of tensile properties with the increase of curing temperature may be due to occurrence of fibre degradation to some extent at higher temperatures [8].

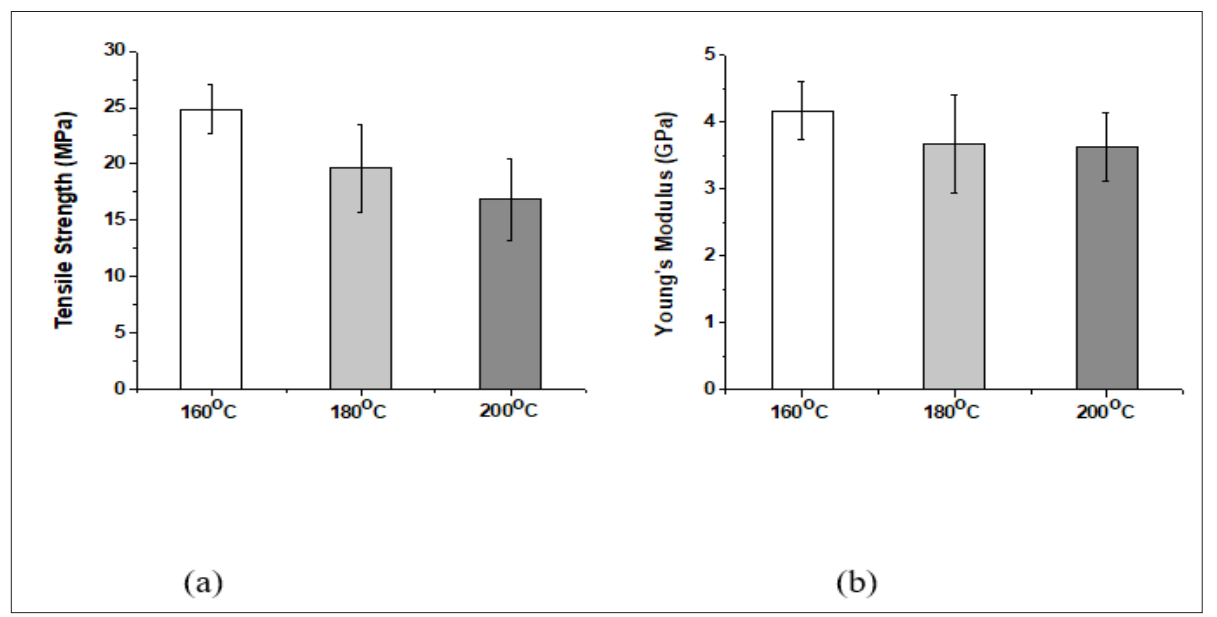

Figure 4: Tensile properties of HAB produced at different curing temperature and time.

HAB produced at different relative humidity of fibre mats: The optimised processing conditions as obtained so far are (i) Acrodur solution to dispersion ratio of $3: 1$, (ii) curing temperature of $160{ }^{\circ} \mathrm{C}$ and (iii) curing time of $20 \mathrm{~min}$. In this section, HAB were produced at different relative humidity of dry, 40, 60 and $80 \%$ using the optimised processing conditions.

From the results of the biocomposites (Figures 5(a)), it can be seen that the average tensile strength did not change significantly for biocomposites produced with dry, $40 \%$ and $60 \%$ RH of hemp fibre mats although $80 \% \mathrm{RH}$ gave a tensile strength reduction of $15 \%$ when compared to dry fibre. However, considering the standard deviation, this $15 \%$ reduction is not statistically significant. Figure 5(b) shows that the average modulus decreased gradually for biocomposites produced with $40 \%, 60 \%$ and $80 \% \mathrm{RH}$ when compared to dry fibre mat biocomposites. This decrease at high RH was relatively small when compared with other binders such as vinylester and unsaturated polyester $[9,10]$. However, the reduction in Young's modulus with the increase of RH is expected since the biocomposites with high RH had comparatively larger deflection (strain) at break. While the dry biocomposites had $1.07 \%$ strain, the biocomposites with $80 \%$ RH had a strain of $3.24 \%$, followed by $3.21 \%$ for the biocomposites produced with $60 \% \mathrm{RH}$ and $2.85 \%$ for $40 \%$ RH.

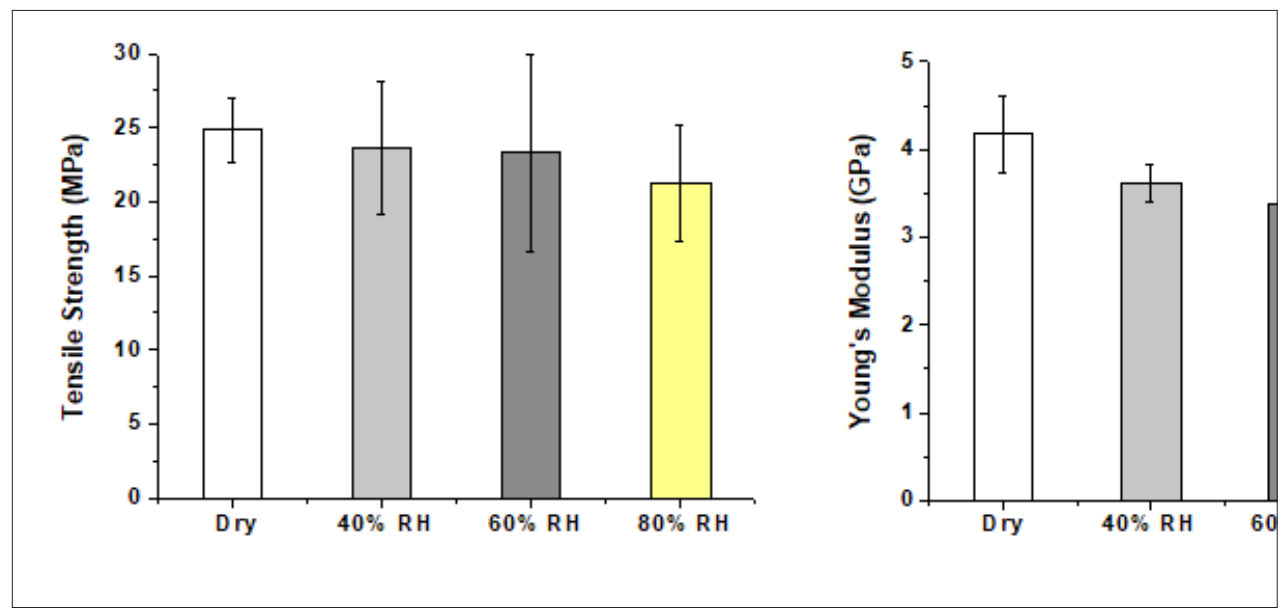

Figure 5: Tensile properties of HAB produced at different relative humidity of hemp fibre mats. 
Curing of acrylic resin in the presence of moisture generally activates the bonding by an esterification reaction between the acid group of acrylic resin and hydroxyl groups of the hemp fibre constituents such as cellulose, hemicelluloses and lignin [11]. The similar tensile strength of the biocomposites produced with dry fibre mat and fibre mats with different relative humidity supports the phenomenon. Considering the above tensile property analysis of the FAB, biocomposites with dry hemp fibre mat at a curing temperature of $160{ }^{\circ} \mathrm{C}$, curing time of $20 \mathrm{~min}$ and Acrodur solution to dispersion ratio of 3:1 was found to be optimum and the rest of the study will be on this biocomposite.

\section{TGA of the hemp fibre, acrodur and HAB}

TGA traces for hemp fibre, Acrodur mixture and HAB produced with optimised condition (curing temperature of $160{ }^{\circ} \mathrm{C}$, curing time of $20 \mathrm{~min}$ and Acrodur solution to dispersion ratio of 3:1) are shown in Figure 6. It can be seen from the thermogram that there are three stages of thermal degradation for all the samples. For the hemp fibre and $\mathrm{HAB}$, the degradation stages are in the temperature range $60-150{ }^{\circ} \mathrm{C}$ (first stage), $150-350{ }^{\circ} \mathrm{C}$ (second stage) and $350-600{ }^{\circ} \mathrm{C}$ (third stage). On the other hand, for Acrodur mixture the degradation stages are in the temperature range of $60-150{ }^{\circ} \mathrm{C}$ (first stage), $150-450{ }^{\circ} \mathrm{C}$ (second stage) and $450-600{ }^{\circ} \mathrm{C}$ (third stage). The three thermal degradation stages are well known in the case of natural cellulosic fibres, where the first degradation stage is due to evaporation of adsorbed moisture, the second degradation stage is due to decomposition of cellulose leading to the formation of volatile products and the third degradation stage is due to oxidation of volatile and charred products [12]. Similarly, the three degradation stages for Acrodur mixture may be due to (i) evaporation of adsorbed moisture, (ii) decomposition of polyester leading to the formation of volatile products and (iii) oxidation of volatile and charred products. At the initial degradation stage, lower loss of weight for the Acrodur mixture compared to the other two samples indicates low moisture adsorption of the hydrophobic cured Acrodur mixture. The greater weight loss at the beginning of the second degradation stage for the cured Acrodur mixture might be due to the decomposition of lower boiling point components (i.e. $\mathrm{CO}, \mathrm{CO}_{2}, \mathrm{CH}_{4}$, ethylene and acetylene) by polyester at $150{ }^{\circ} \mathrm{C}$ to 330 ${ }^{\circ} \mathrm{C}$ [13].

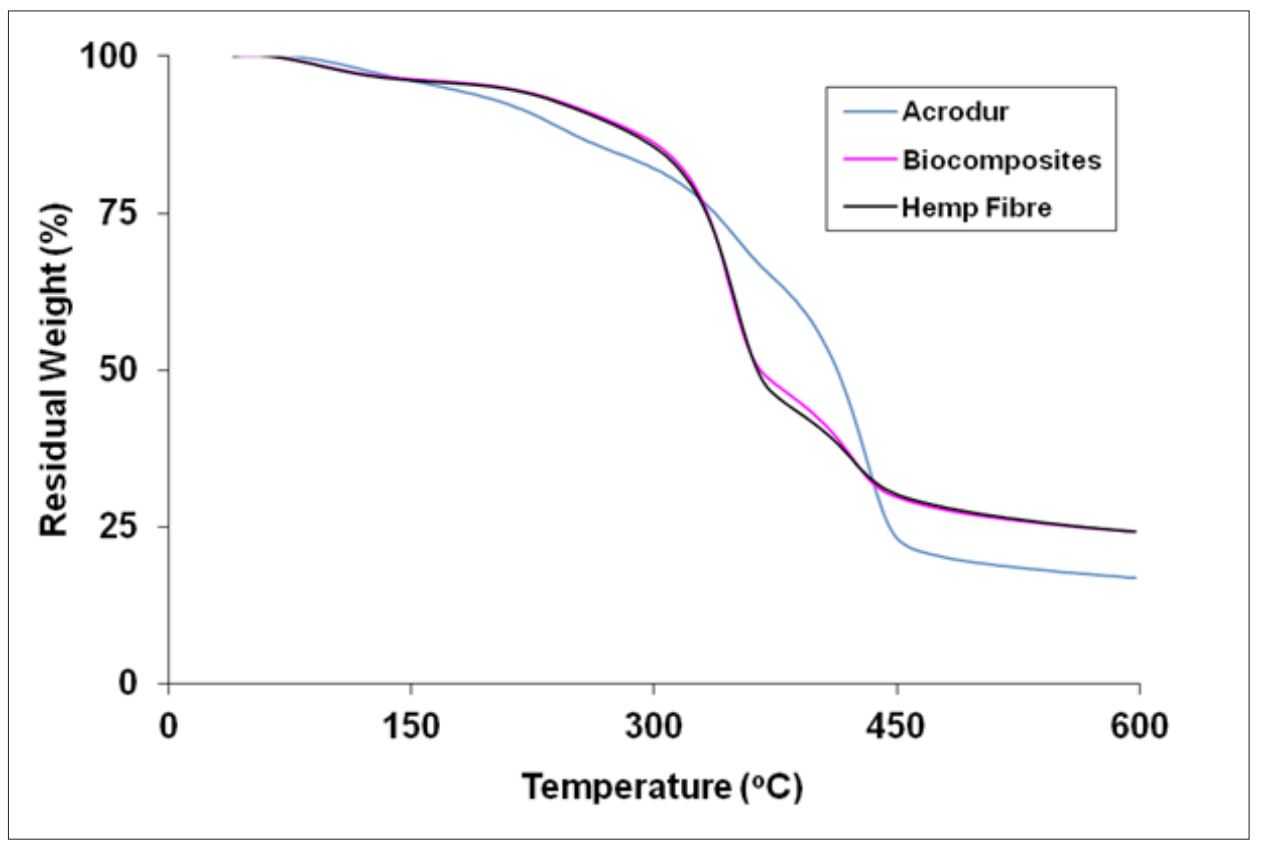

Figure 6: TGA thermograms of hemp fibre, Acrodur and HAB.

It can be seen from the figure that at all stages of thermal degradation, the weight losses are almost similar for hemp fibre and HAB. Therefore, it can be conferred that production of biocomposites using Acrodur mixture as matrix does not affect the thermal stability of the biocomposites. However, the higher residual weight for the hemp fibre and biocomposites compared to that of Acrodur mixture supports the formation of lignicellulose complex at higher temperature of the natural fibres [14].

Contact angle measurement of hemp fibre, acrodur and HAB
Figure 7 shows the contact angle photographs of hemp fibre, Acrodur and $\mathrm{HAB}$ produced with optimised condition (curing temperature of $160{ }^{\circ} \mathrm{C}$, curing time of $20 \mathrm{~min}$ and Acrodur solution to dispersion ratio of $3: 1$ ). Table 1 shows the average contact angle of the samples. It can be seen from the table that the highest contact angle was found in the cured Acrodur mixture followed by HAB and finally by the hemp fibre. The increased contact angle (hydrophobicity) of the biocomposites compared to the hemp fibre may be attributed to the fibre matrix bonding at the interface to some extent. 


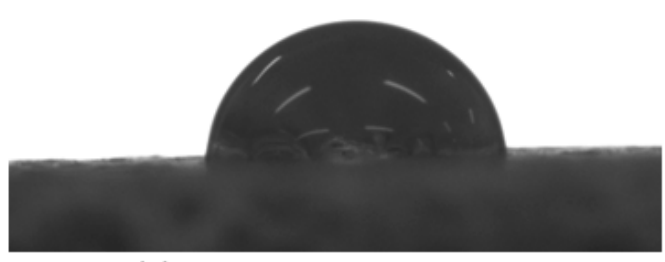

(a)

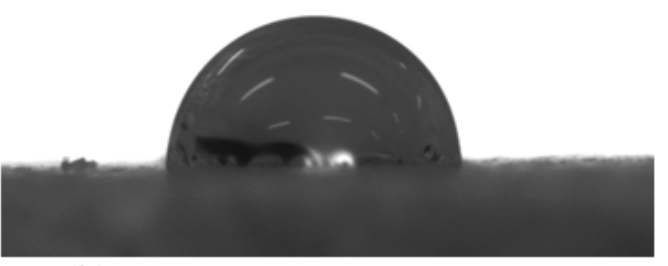

(b)

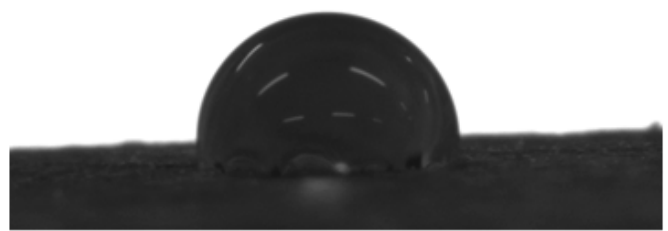

(c)

Figure 7: Contact angle photographs of hemp, cured Acrodur and HAB with optimized processing conditions.

Table 1: Average contact angle of the samples.

\begin{tabular}{|c|c|}
\hline Sample & Contact Angle $\left({ }^{\circ}\right)$ \\
\hline Hemp Fibre & $90.6 \pm 0.52$ \\
\hline Cured Acrodur Mixture & $93.6 \pm 0.13$ \\
\hline Biocomposite & $93 \pm 0.10$ \\
\hline
\end{tabular}

\section{Water immersion tests}

Figure 8 shows the water uptake of the biocomposites produced with optimised condition (curing temperature of $160{ }^{\circ} \mathrm{C}$, curing time of $20 \mathrm{~min}$ and Acrodur solution to dispersion ratio of 3:1) with immersion time. The percentage water uptake increased logarithmically with the increase of water immersion time and the percentage of water uptake is about $10 \%$ at the end of four-week immersion. The high amount of water uptake of the biocomposites indicates poor fibre-matrix adhesion due to the reasons described below.

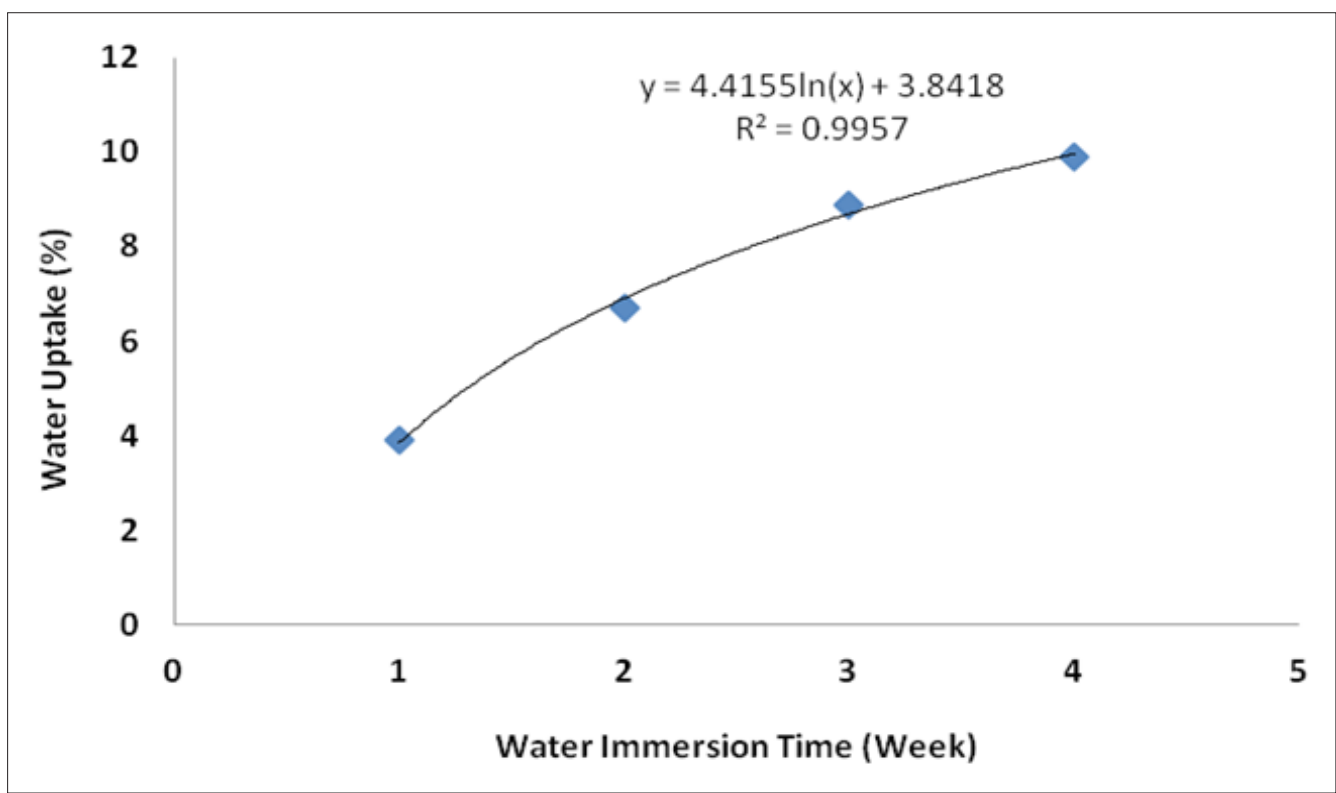

Figure 8: Water uptake versus water immersion time.

Table 2 shows the tensile strength and Young's modulus of control biocomposites (no water immersion) compared to the biocomposites after water immersion periods of up to four weeks at room temperature. It can be seen that after 1 week of immersion the biocomposites tensile strength was found to be reduced by $30.2 \%$ and the immersion of the biocomposites for further periods of time (2, 3 and 4 weeks) did not bring any significant amount of strength loss. Since the cured Acrodur mixture is porous (Figure 
9), the biocomposite produced had porous structure (Figure 10(a)) which might allow the water to be wicked easily and damaged the fibre-matrix interfacial bonding by swelling. Loss of fibre-matrix integrity can be seen by larger pores and fibre separations of the optical picture of water immersed biocomposite (Figure 10(b)). Further immersion of the biocomposites for longer period did not produce any significant reduction in strength which may be due to an equilibrium state was reached. In the case of good fibre matrix adhesion the path of wicking of the water molecules is believed to be tortuous due to better fibre-matrix integrity while the wicking path would be nearly straight for weak fibre matrix bonding, as depicted in Figure 11. In case of the current study, the straight path of wicking of water molecules may accelerate the degradation of fibre matrix bond integrity.
Table 2: Tensile properties of $\mathrm{HAB}$ with water soaking time.

\begin{tabular}{|c|c|c|}
\hline Sample & Tensile Strength (MPa) & Young's Modulus (GPa) \\
\hline Control & $24.8 \pm 2.16$ & $4.2 \pm 0.44$ \\
\hline 1 week & $17.3 \pm 1.63$ & $3.4 \pm 0.42$ \\
\hline 2 weeks & $17.0 \pm 4.16$ & $2.4 \pm 0.40$ \\
\hline 3 weeks & $15.5 \pm 2.42$ & $2.1 \pm 0.19$ \\
\hline 4 weeks & $14.0 \pm 2.80$ & $1.8 \pm 0.26$ \\
\hline
\end{tabular}

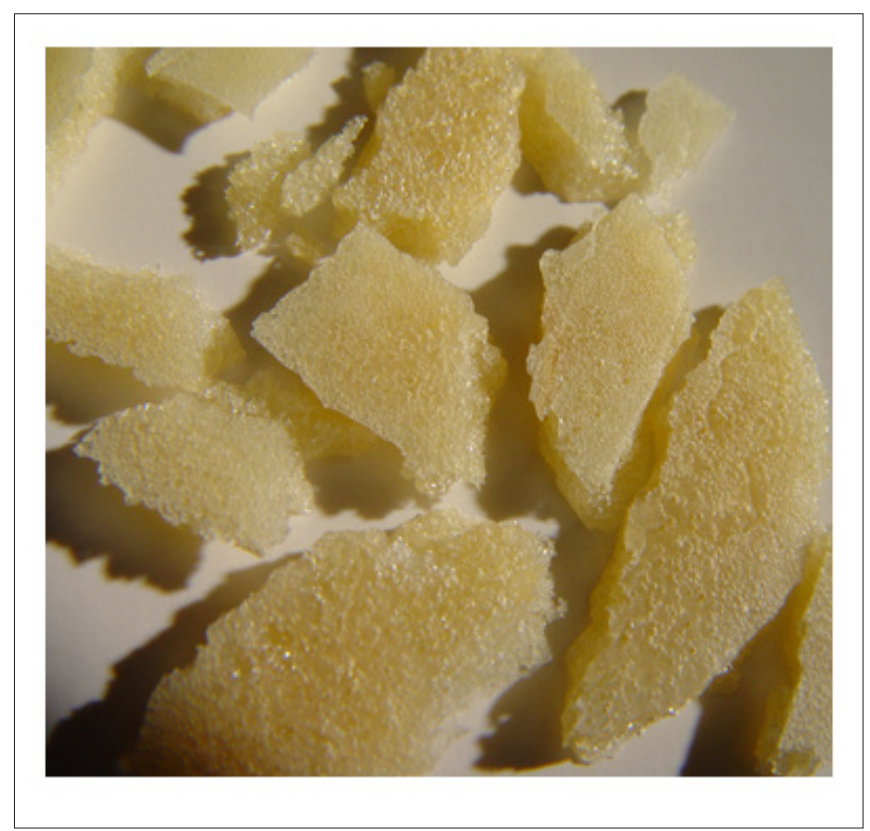

Figure 9: Porous structure of cured Acrodur mixture.

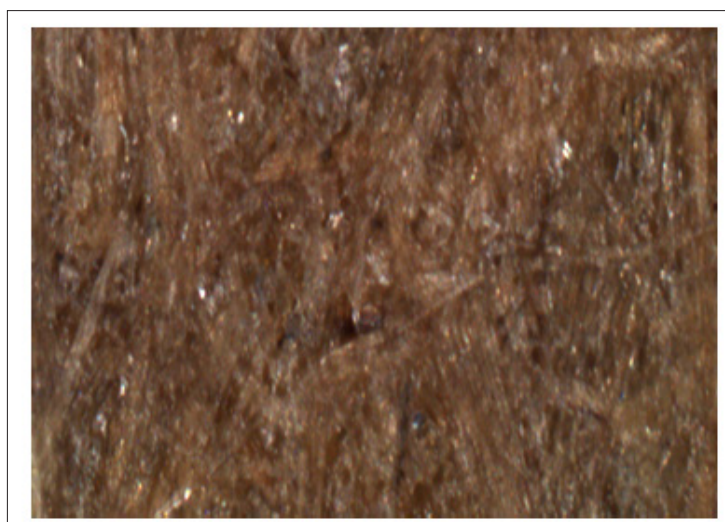

(a)

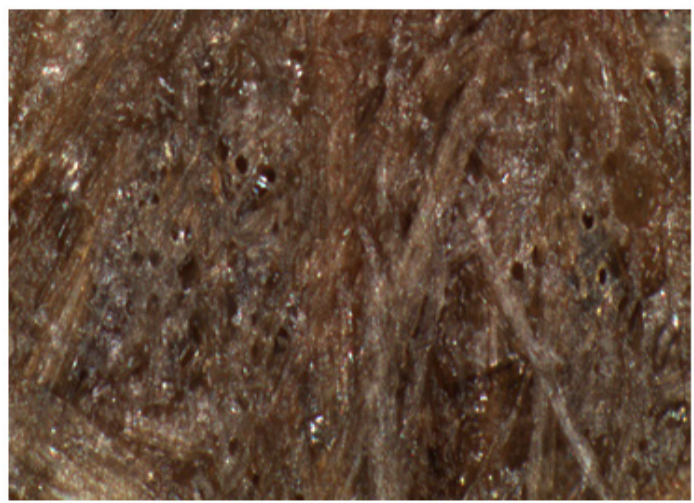

(b)

Figure 10: Optical micrographs of biocomposites showing loss of fibre fibre-matrix structural integrity (A. control and B. 1 week water immersion). 


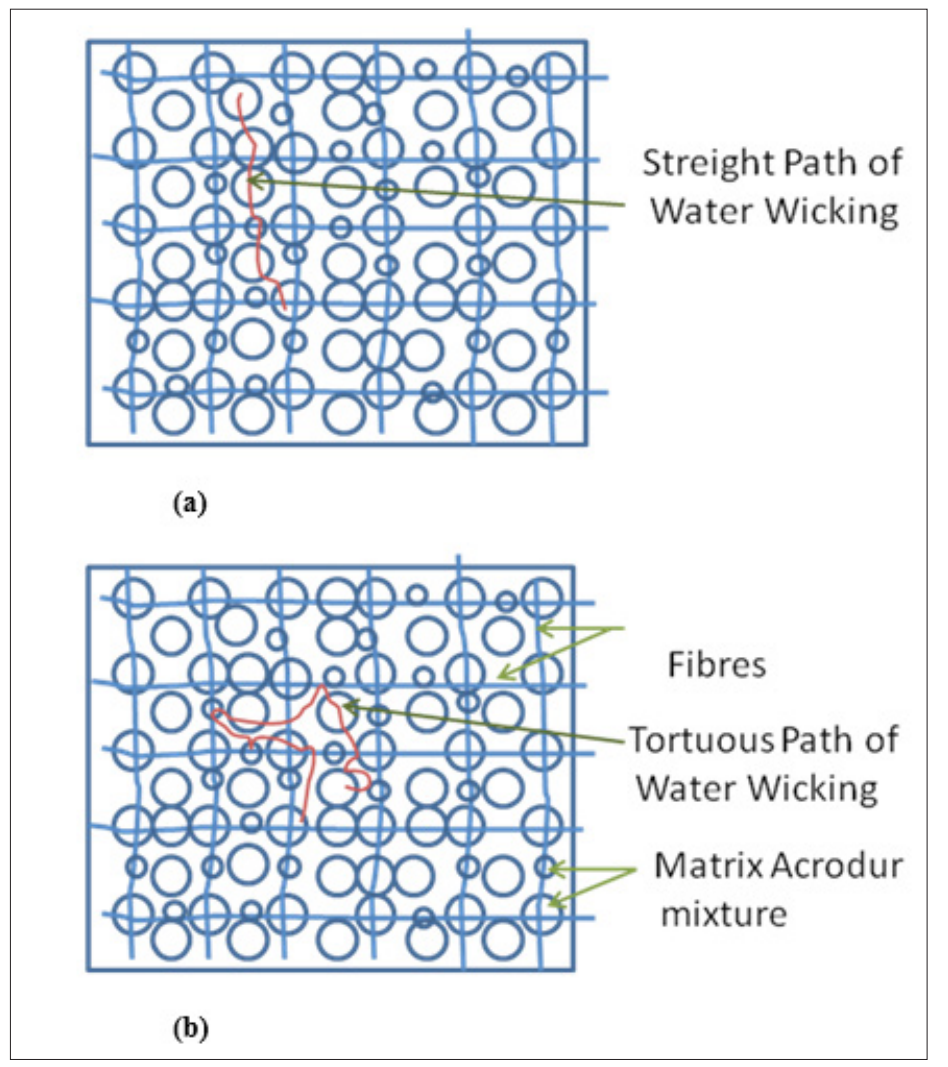

Figure 11: The mechanism of wicking of water in biocomposites (a) in the case of weak fibre matrix bonding and (b) in the case of good fibre matrix bonding.

From Table 2 it can also be seen that after one-week immersion, Young's modulus of the biocomposite decreased by about $19 \%$ when compared to the control sample (zero week or no immersion). The decrease of Young's modulus was $29.4 \%$ after 2-week immersion from that of 1-week immersion. Further immersion of the biocomposites after 2 weeks (for 3 and 4 weeks) was found to be slow. The reduction of Young's modulus could be due to plasticization of the biocomposites by the adsorbed water molecules.

\section{Conclusion}

HAB produced with Acrodur resin have good specific tensile properties. The thermal resistance of the biocomposites appeared to be similar to the fibres and water resistance was poor. Therefore, the biocomposites produced in the current study can find their application in indoors only. Since moisture content in the fibres does not significantly affect the biocomposites production using Acrodur resin unlike other conventional resins, drying of the preforms may be minimised or even eliminated. On top of that Acrodur would be very environmentally friendly as it does not emit any poisonous gases harmful for the living beings. As the changing of the processing variables varied the biocomposite tensile properties, these processing variables can be tailored to suit a wide variety of applications. High loss of tensile properties upon water immersion testing suggested straight path of wicking of water into the biocomposites due to weak fibre-matrix interface.

\section{Acknowledgement}

The authors are thankful to CRC-ACS, Australia, for supporting this project.

\section{References}

1. Saheb DN, Jog JP (1999) Natural fiber polymer composites: A review. Advances in Polymer Technology 18(4): 351-363.

2. Thomsen AB, Bohn V, Nielsen K, Pallesen B, Orgensen MS (2000) Bioresource Hemp 13-16: 1-6

3. US Patent 5993709 (1999) Method for making composite board using phenol formaldehyde binder.

4. Young NB, Kim MG (2007) Evaluation of melamine-modified ureaformaldehyde resins as particleboard binders. J Appl Polym Sci 106(6): 4148-4156.

5. State of California Environmental Protection Agency, office of environmental health hazard assessment (2006) Safe drinking water and toxic enforcement act of 1986 - Chemicals known to the state to cause cancer or reproductive toxicity.

6. International Agency for Research on Cancer (IARC). Monographs on evaluation of carcinogenic risks for humans- formaldehyde.

7. Prioritization of Toxic Air Contaminants (2001) Children's environmental health protection act. Formaldehyde, OEHHA, USA.

8. Kalia S, Kaith BS, Kaur I (2009) Pretreatments of natural fibers and their application as reinforcing material in polymer biocomposites-A review. 
Polym Eng Sci 49(7): 1253-1272.

9. Chen H, Miao M, Ding X (2011) Chemical treatments of bamboo to modify its moisture absorption and adhesion to vinyl ester resin in humid environment. J Compos Mater 45(14): 1533-1542.

10. Miao M, Milanovic N, Shen SZ (2011) Effect of moisture exposure during fabrication on flax/polyester biocomposites. ICCM.

11. Reck B, Türk J (1999) Thermally curable aqueous acrylic resins-a new class of duroplastic binders for wood and natural fibers. Die Angewandte Makromolekulare Chemie 272(1): 5-10.
12. Ouajai S, Shanks RA (2005) Composition, structure and thermal degradation of hemp cellulose after chemical treatments. Polym Degrad and Stabil 89: 327-335.

13. Emil B, Barbara CL (1986) Polyesters: A review of the literature on products of combustion and toxicity. Fire and Materials 10(3-4): 107123.

14. Islam MS, Pickering KL, Foreman NJ (2011) Influence of alkali fiber treatment and fiber processing on the mechanical properties of hemp $\backslash$ epoxy biocomposites. J Appl Polym Sci 119(6): 3696-3707. 